\title{
Issues in the Modeling and Boundary Control of 2D Heat Flow: POD Based Modeling
}

\author{
Mehmet Önder Efe
}

\begin{abstract}
Low Dimensional (LD) modeling of systems governed by Partial Differential Equations (PDE) has been studied several times in the past. Various types of boundary excitations have been considered. This paper demonstrates how the external stimuli is made explicit in an autonomous set of ODEs and how the excitations along nonpoint subdomains of the boundaries are handled. Dirichlét type boundary excitations are considered and 2D heat equation has been chosen as the test bed. Linearity of the system makes it a good choice for investigating the stability and performance issues. Proper Orthogonal Decomposition (POD) is used in the modeling stage and it is shown that the developed model reconstructs the essential dynamics of the solution of the PDE successfully. The contributions of the paper are on the effects of the number of modes on the model performance, spectral dependence of LD models to the initial and boundary conditions and the prime importance of a fundamental assumption.
\end{abstract}

\section{INTRODUCTION}

Modeling and control of PDE processes is an interesting research area and the outcomes of which address many physical phenomena displaying spatial continuity, e.g. heat and fluid flows. It is a well known fact that for linear PDEs, there are well established alternatives other than POD, (Gügercin 2000), but POD is a widely used method in modeling of more complicated systems. The goal of this paper is to present a model reduction with a discussion on the effect of parameters entering into the POD algorithm.

Proper Orthogonal Decomposition was proposed in the pioneering work of Lumley (1967) with the goal of unfolding the modal nature of turbulent flows. Sirovich (1987) introduced the method of snapshots for reducing the computational intensity of the original POD algorithm. The POD method is widely accepted as a powerful tool for decomposing the content of a time varying spatially continuous process into the spatial and temporal constituents. The spatial part is a set of basis functions while the temporal part is a set of differential equations. The decomposition is accomplished in the order of dominance, which is a significant property enabling the designer truncate the expression at a particular mode number. Modeling of flow problems governed by PDEs have therefore enjoyed the POD method in obtaining the finite dimensional models at the cost of giving concessions from the model performance, see for example Rowley et al (2004, 2005), Ly and Tran (2001), Caraballo et al (2004) and the references therein.

Procedurally, the PDE set is solved for the given initial and boundary conditions. Several samples from the solution set are selected and the POD method with Galerkin projection is applied. As a result of this, a set of autonomous Ordinary Differential Equations (ODEs) is obtained. The solution of the obtained ODEs with the given initial conditions synthesize the temporal part of the solution and the spatial basis functions obtained through the POD method yields the approximate solution of the PDE. Unfortunately, the set of ODEs are specific to the initial and boundary

This work was supported by TOBB Economics and Technology University, BAP Program, under contract no ETÜ-BAP-2006/04

Dr. Efe is with TOBB Economics and Technology University, Department of Electrical and Electronics Engineering, Sögütözü, Ankara, Turkey, Phone: +90-312-292-4064, Fax: +90-312-292 4091 onderefedieee. org conditions used in the model derivation stage. In other words, one needs to change the ODE model for every different instance of boundary excitation regimes. This paper demonstrates how the aforementioned autonomous set of ODEs are made non-autonomous and external excitations are seen explicitly in the model. Aside from making the boundary excitations explicit in the model, we demonstrate that the validity of the developed model is extended to a set of boundary conditions, which constitute the final stage of the modeling effort. The prominent feature of the approach presented is that the algorithm yields a model using some boundary excitations, but the model maintains its validity for different but similar signals. The modeling procedure is followed with pointwise boundary excitations for 1D Burgers equation in Efe (2004) and 2D heat equation in Efe (2003). This paper extends the boundary excitations to nonpoint subdomains of the boundaries, which results in much richer heat distributions in the snapshots than those obtained in Efe (2003).

Another variation of the LD modeling of $2 \mathrm{D}$ heat flow problem has been taken into consideration in Atwell and King (2001), which consider a modified 2D heat transport problem with control input explicitly available in the PDE. The thermal diffusivity parameter has been taken as a known constant and several control strategies have been assessed with the modeling results of POD approach. Clearly, the availability of the control input in the PDE means that the excitation is not only through the boundaries, and the obtained low order model would have the excitation input(s) explicitly.

This paper is organized as follows. The second section summarizes the POD algorithm specific to the modeling of $2 \mathrm{D}$ heat flow problem. In the third section, development of the reduced order model for the 2D heat flow is analyzed. The fourth section presents the modeling results with an emphasis on the spectral dependence of the model to the operating conditions initial and boundary. The last section summarizes the contributions of the paper.

\section{Proper Orthogonal Decomposition}

Consider the ensemble $U_{i}(x, y), i=1,2, \ldots, N_{s}$, where $N_{s}$ is the number of elements. Every element of this set corresponds to a snapshot observed from a process, say for example 2D heat flow with initial and boundary conditions,

$$
\begin{aligned}
u_{t}(x, y, t) & =c^{2}\left(u_{x x}(x, y, t)+u_{y y}(x, y, t)\right) \\
u(x, 0, t) & =f_{1}(x) \gamma_{1}(t), u(1, y, t)=f_{2}(y) \gamma_{2}(t), \\
u(x, 1, t) & =f_{3}(x) \gamma_{3}(t), u(0, y, t)=f_{4}(y) \gamma_{4}(t), \\
u(x, y, 0) & =0 \forall(x, y),
\end{aligned}
$$

where, $c$ is the known constant thermal diffusivity parameter, and the subscripts $x, y$ and $t$ refer to the partial differentiation with respect to $x, y$ and time, respectively. The continuous time process takes place over the physical domain $\Omega:=\{(x, y) \mid(x, y) \in[0,1] \times$ $[0,1]\}$ and the solution is obtained on a spatial grid denoted by $\Omega_{d}$, which describes the coordinates of the pixels of every snapshot in the ensemble. The entities described over $\Omega_{d}$ are matrices in $\mathbb{R}^{N_{y} \times N_{x}}$. The goal is to find an orthonormal basis set letting us to write the solution as 


$$
u(x, y, t)=\sum_{i=1}^{R_{L}} \alpha_{i}(t) \Phi_{i}(x, y),
$$

where $\alpha_{i}(t)$ is the $i^{\text {th }}$ temporal mode, $\Phi_{i}(x, y)$ is the $i^{\text {th }}$ spatial function (basis functions or the eigenfunctions), $R_{L}$ is the number of independent basis functions that can be synthesized from the given ensemble, or equivalently that spans the space described by the ensemble. It will later be clear that if the basis set $\left\{\Phi_{i}(x, y)\right\}_{i=1}^{R_{L}}$ is an orthonormal set, Galerkin projection yields the autonomous ODEs directly. Let us summarize the POD procedure.

Step 1. Start calculating the $N_{s} \times N_{s}$ dimensional correlation matrix $L$, the $(i j)^{\text {th }}$ entry of which is $L_{i j}:=\left\langle U_{i}, U_{j}\right\rangle_{\Omega_{d}}$, where $\langle., .\rangle_{\Omega_{d}}$ is the inner product operator defined over $\mathbb{R}^{N_{y} \times N_{x}}$.

Step 2. Find the eigenvectors (denoted by $v_{i}$ ) and the associated eigenvalues $\left(\lambda_{i}\right)$ of the matrix $L$. Sort them in a descending order in terms of the magnitudes of $\lambda_{i}$. Note that every $v_{i}$ is an $N_{s} \times 1$ dimensional vector satisfying $v_{i}^{\mathrm{T}} v_{i}=\frac{1}{\lambda_{i}}$, here, for simplicity of the exposition, we assume that the eigenvalues are distinct.

Step 3. Construct the basis set by using

$$
\Phi_{i}(x, y)=\sum_{j=1}^{N_{s}} v_{i j} U_{j}(x, y)
$$

where $v_{i j}$ is the $j^{\text {th }}$ entry of the eigenvector $v_{i}$, and $i=$ $1,2, \ldots, R_{L}$, where $R_{L}=\operatorname{rank}(L)$. It can be shown that $\left\langle\Phi_{i}(x, y), \Phi_{j}(x, y)\right\rangle_{\Omega}=\delta_{i j}$ with $\delta_{i j}$ being the Kronecker delta function. Notice that the basis functions are admixtures of the snapshots, (Ly and Tran 2001, Efe and Özbay 2003).

Step 4. Calculate the temporal coefficients. Taking the inner product of both sides of (2) with $\Phi_{i}(x, y)$, the orthonormality property leads to

$$
\begin{aligned}
\alpha_{i}\left(t_{0}\right) & =\left\langle\Phi_{i}(x, y), u\left(x, y, t_{0}\right)\right\rangle_{\Omega} \\
& =\left\langle\phi_{i}, U_{t_{0}}\right\rangle_{\Omega_{d}} \\
& :=\frac{1}{N_{s}} \sum_{l=1}^{N_{x}} \sum_{j=1}^{N_{y}} \phi_{i}\left(x_{l}, y_{j}\right) U_{t_{0}}\left(x_{l}, y_{j}\right) \\
& =\phi_{i} \star U_{t_{0}},
\end{aligned}
$$

where $\phi_{i} \in \mathbb{R}^{N_{y} \times N_{x}}$ is a sampled form of the basis functions $\Phi_{i}$ defined over $\Omega$. The operator denoted by $\star$ computes a real number that is the sum of all elements of a matrix obtained through the elementwise multiplication of the two matrices that $\star$ lies in between. Without loss of generality, an element of the ensemble $\left\{U_{i}(x, y)\right\}_{i=1}^{N_{s}}$ may be $U\left(x, y, t_{0}\right)$. Therefore, in order to generate the temporal gain, $\alpha_{k}(t)$, of the spatial eigenfunction $\phi_{k}$, one would take the inner product of $\phi_{k}$ with the elements of the ensemble as given below,

$$
\begin{aligned}
\left\langle U_{1}, \phi_{k}\right\rangle_{\Omega_{d}} & \approx \alpha_{k}\left(t_{1}\right) \\
\left\langle U_{2}, \phi_{k}\right\rangle_{\Omega_{d}} & \approx \alpha_{k}\left(t_{2}\right) \\
& \vdots \\
\left\langle U_{N_{s}}, \phi_{k}\right\rangle_{\Omega_{d}} & \approx \alpha_{k}\left(t_{N_{s}}\right) .
\end{aligned}
$$

Note that the temporal coefficients satisfy orthogonality properties over the discrete set $t \in\left\{t_{1}, t_{2}, \ldots, t_{N_{s}}\right\}$ (See (6)). For a more detailed discussion on the POD method, the reader is referred to (Lumley 1967, Ly and Tran 2001, Efe and Özbay 2003-2004, Caraballo et al 2004, Rowley et al 2004, Rowley 2005) and the references therein,

$$
\sum_{i=1}^{N_{s}}\left\langle U_{i}(x, y), \Phi_{k}(x, y)\right\rangle_{\Omega_{d}}^{2} \approx \sum_{i=1}^{N_{s}} \alpha_{i}^{2}\left(t_{i}\right)=\lambda_{k}
$$

Fundamental Assumption: The majority of works dealing with POD and model reduction applications presume that the flow is dominated by coherent modes. Because of the dominance of coherent modes, the typical spread of the eigenvalues of the correlation matrix turns out to be logarithmic and the terms decay very rapidly in magnitude. This fact enables us to assume that a reduced order representation, say with $M$ modes $\left(M<R_{L}\right)$ can also be written as an equality

$$
u(x, y, t)=\sum_{i=1}^{M} \alpha_{i}(t) \Phi_{i}(x, y),
$$

and the reduced order model is derived under the assumption that (7) satisfies the governing PDE in (1), (Ravindran 2000, Ly and Tran 2001, Efe and Özbay 2003, Efe and Özbay 2004, Caraballo et al 2004). Unsurprisingly, such an assumption results in a model having uncertainties, however, one should keep in mind that the goal is to find a model, which matches the infinite dimensional system in some sense of approximation with typically $M \ll R_{L} \leq N_{s}$. To represent how good such an expansion is, a percent energy measure is defined as follows

$$
E=100 \frac{\sum_{i=1}^{M} \lambda_{i}}{\sum_{i=1}^{R_{L}} \lambda_{i}},
$$

where the tendency of $E \rightarrow 100 \%$ means that the model captures the dynamical information contained in the snapshots well. Conversely, an insufficient model will be obtained if $E$ is far below $100 \%$. In the next section, we demonstrate how the boundary condition is transformed to an explicit control input in the ODEs.

\section{REDUCED ORder MOdELING}

In the order reduction phase, we need to obtain the autonomous ODE model first. Towards this goal, if (7) is a solution to the PDE in (1), then it has to satisfy the PDE. Substituting (2) into (1) with the fundamental assumption yields

$$
\sum_{i=1}^{M} \dot{\alpha}_{i}(t) \Phi_{i}(x, y)=c^{2} \sum_{i=1}^{M} \alpha_{i}(t) \Psi_{i}(x, y),
$$

where $\Psi_{i}(x, y)=\frac{\partial^{2} \Phi_{i}(x, y)}{\partial x^{2}}+\frac{\partial^{2} \Phi_{i}(x, y)}{\partial y^{2}}$. Taking the inner product of both sides with $\Phi_{k}(x, y)$ and remembering $\left\langle\Phi_{i}(x, y), \Phi_{k}(x, y)\right\rangle_{\Omega}=\delta_{i k}$ with $\delta_{i k}$ being Kronecker delta yields

$$
\dot{\alpha}_{k}(t)=c^{2} \sum_{i=1}^{M} \alpha_{i}(t)\left\langle\Phi_{k}(x, y), \Psi_{i}(x, y)\right\rangle_{\Omega},
$$

Defining $\zeta_{k}$ as the entity in $\Omega_{d}$ corresponding to the entity $\Psi_{k}$ in $\Omega$, one could rewrite (10) as

$$
\dot{\alpha}_{k}(t)=c^{2} \sum_{i=1}^{M} \alpha_{i}(t)\left\langle\phi_{k}, \zeta_{i}\right\rangle_{\Omega_{d}} .
$$

The equation in (11) can be written explicitly by using $\star$ operator as

$$
\dot{\alpha}_{k}(t)=c^{2} \sum_{i=1}^{M} \alpha_{i}(t)\left(\phi_{k}(x, y) \star \zeta_{i}(x, y)\right)
$$

Notice that $\star$ operator can be applied over nonoverlapping subdomains of $\Omega_{d}$. This lets us separate the entries corresponding to boundaries without modifying the values of $\phi_{k}(x, y) \star \zeta_{i}(x, y)$ as seen in (13), 


$$
\begin{aligned}
\dot{\alpha}_{k}(t)=\quad & c^{2} \sum_{i=1}^{M} \alpha_{i}(t)\left(\phi_{k}(x, 0) \star \zeta_{i}(x, 0)+\phi_{k}(1, y) \star \zeta_{i}(1, y)+\right. \\
& \left.\phi_{k}(x, 1) \star \zeta_{i}(x, 1)+\phi_{k}(0, y) \star \zeta_{i}(0, y)\right)+ \\
& c^{2} \sum_{i=1}^{M} \alpha_{i}(t)\left(\phi_{k}^{\circ}(x, y) \star \zeta_{i}^{\circ}(x, y)\right) .
\end{aligned}
$$

In above, $\phi_{k}^{\circ}(x, y)$ denotes a matrix that is obtained when the boundary elements of $\phi_{k}(x, y)$ are removed. The $k^{\text {th }}$ component of the first summation above, which is obtained when $i=k$, can be separated from the expression and we obtain (14), which let us embed the boundary conditions into the expression,

$$
\begin{aligned}
& \dot{\alpha}_{k}(t)=c^{2} \alpha_{k}(t)\left(\phi_{k}(x, 0) \star \zeta_{k}(x, 0)+\phi_{k}(1, y) \star \zeta_{k}(1, y)+\right. \\
& \left.\phi_{k}(x, 1) \star \zeta_{k}(x, 1)+\phi_{k}(0, y) \star \zeta_{k}(0, y)\right)+ \\
& c^{2} \sum_{i=1}^{M} \alpha_{i}(t)\left(1-\delta_{i k}\right)\left(\phi_{k}(x, 0) \star \zeta_{i}(x, 0)+\right. \\
& \phi_{k}(1, y) \star \zeta_{i}(1, y)+\phi_{k}(x, 1) \star \zeta_{i}(x, 1)+ \\
& \left.\phi_{k}(0, y) \star \zeta_{i}(0, y)\right)+c^{2} \sum_{i=1}^{M} \alpha_{i}(t)\left(\phi_{k}^{\circ}(x, y) \star \zeta_{i}^{\circ}(x, y)\right) .
\end{aligned}
$$

At this stage of the modeling, we need to paraphrase the boundary conditions in such a way that the final expression above can be incorporated with these conditions. The underlying idea is straightforward: If (7) is a solution, then is must be satisfied at the boundaries as well. This lets us write the following

$$
\sum_{i=1}^{M} \alpha_{i}(t) \phi_{i}(x, 0)=f_{1}(x) \gamma_{1}(t)
$$

which can be paraphrased as

$$
\alpha_{k}(t) \phi_{k}(x, 0)=f_{1}(x) \gamma_{1}(t)-\sum_{i=1}^{M}\left(1-\delta_{i k}\right) \alpha_{i}(t) \phi_{i}(x, 0) .
$$

The expression above can be inserted into the first line of (14) and we explicitly see $\gamma_{1}(t)$ in our expression. Likewise, repeating the same arrangements for the other three edges (the boundaries), we end up with

$$
\begin{aligned}
\dot{\alpha}_{k}(t)= & c^{2}\left(f_{1}(x) \star \zeta_{k}(x, 0)\right) \gamma_{1}(t)+ \\
& c^{2}\left(f_{2}(y) \star \zeta_{k}(1, y)\right) \gamma_{2}(t)+ \\
& c^{2}\left(f_{3}(x) \star \zeta_{k}(x, 1)\right) \gamma_{3}(t)+ \\
& c^{2}\left(f_{4}(y) \star \zeta_{k}(0, y)\right) \gamma_{4}(t)+ \\
& c^{2} \sum_{i=1}^{M} \alpha_{i}(t)\left(\phi_{k} \star \zeta_{i}\right. \\
& -\phi_{i}(x, 0) \star \zeta_{k}(x, 0)-\phi_{i}(1, y) \star \zeta_{k}(1, y) \\
& \left.-\phi_{i}(x, 1) \star \zeta_{k}(x, 1)-\phi_{i}(0, y) \star \zeta_{k}(0, y)\right)
\end{aligned}
$$

which can be written compactly as

$$
\dot{\alpha}(t)=A \alpha(t)+B \Gamma(t)
$$

where $\alpha(t)=\left(\begin{array}{llll}\alpha_{1}(t) & \alpha_{2}(t) & \ldots & \alpha_{M}(t)\end{array}\right)^{\mathrm{T}}, \quad \Gamma(t)=$ $\left(\gamma_{1}(t) \gamma_{2}(t) \gamma_{3}(t) \gamma_{4}(t)\right)^{\mathrm{T}}$ and

$$
\begin{aligned}
A_{k i}= & c^{2}\left(\phi_{k}(x, y) \star \zeta_{i}(x, y)-\phi_{i}(x, 0) \star \zeta_{k}(x, 0)\right. \\
& -\phi_{i}(1, y) \star \zeta_{k}(1, y)-\phi_{i}(x, 1) \star \zeta_{k}(x, 1) \\
& \left.-\phi_{i}(0, y) \star \zeta_{k}(0, y)\right)
\end{aligned}
$$

and the $k^{\text {th }}$ row of the input matrix is

$$
\begin{aligned}
B_{k}= & c^{2}\left(f_{1}(x) \star \zeta_{k}(x, 0)\left|f_{2}(y) \star \zeta_{k}(1, y)\right| f_{3}(x) \star \zeta_{k}(x, 1) \mid\right. \\
& \left.f_{4}(y) \star \zeta_{k}(0, y)\right)
\end{aligned}
$$

This result practically lets us have a linear dynamical model for the infinite dimensional process in (1), which is aimed to be controlled through the boundaries.

\section{Modeling Results}

According to the described procedure, several tests have been done. Due to the numerical advantages, the PDE has been solved by using Crank-Nicholson method (See Farlow (1993) for details), with a step size of $1 \mathrm{msec}$. The initial thermal distribution is taken zero everywhere and the thermal diffusivity constant is set as $c=1$. In order to form the solution, a linear grid having $N_{x}=N_{y}=25$ points in $x$-direction and $y$-direction respectively. According to the above parameter values, a total of 501 snapshots embody the entire numerical solution, among which a linearly sampled $N=251$ snapshots have been used for the POD scheme. Although one may use the entire set of snapshots, it has been shown by Sirovich (1987) that a reasonably descriptive subset of them can be used for the same purpose. In the literature, this approach is called method of snapshots, which significantly reduce the computational intensity of the overall scheme, (See also Ravindran 2000 and Ly and Tran 2001). Once the modes have been obtained, we truncated the solution at $M=8$, which represents $\% 99.6563$ of the total energy described by (8).

In order to demonstrate the performance of the dynamic model, we have set the functions that are effective along the boundaries as $f_{1}(x)=f_{3}(x)=\sin (2 \pi x)$ and $f_{2}(y)=f_{4}(y)=\sin (2 \pi y)$. As the temporal excitations we chose the following input signals,

$$
\begin{aligned}
& \gamma_{1}(t)=\sin (2 \pi 80 t(T / 2-t)) \\
& \gamma_{2}(t)=\sin (2 \pi 110 t(T-t), \\
& \gamma_{3}(t)=\sin (2 \pi 100 t(T / 3-t)), \\
& \gamma_{4}(t)=\sin (2 \pi 110 t(T-t),
\end{aligned}
$$

where $T=0.5$ seconds. The choice of the above excitations signals is deliberate as they are spectrally rich. As can be seen from Figure $1, \alpha_{k}(t)$ 's will undergo regimes that change sometimes slowly and sometimes fast depending on the spectral composition of the external inputs. Under these conditions, the results obtained in time domain are illustrated in Figure 2.

Undoubtedly, one would expect a good match between the state variables obtained from the POD algorithm and the state variables obtained through the numerical solution of the ODE set in (18). One might question whether the model is specific to the boundary conditions above. Remedying this is accomplished by choosing another set of external inputs and obtaining the response of the model without modifying the model parameters. For this purpose, we set 

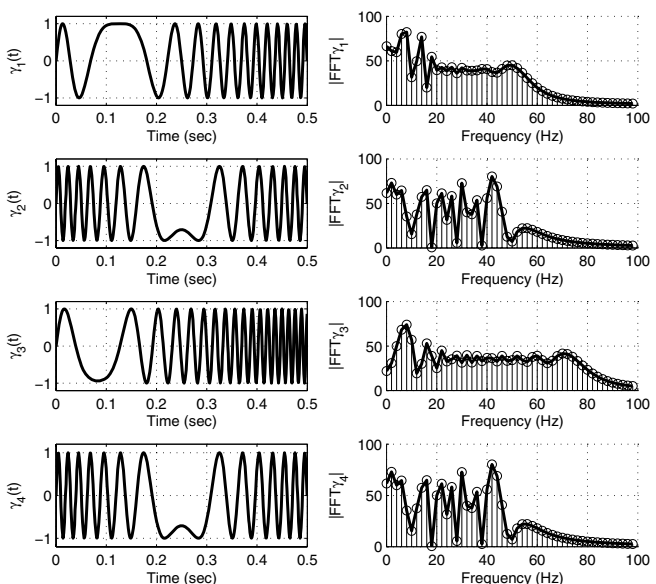

Fig. 1. Temporal and spectral views of the boundary excitations used in the derivation of the low order model.
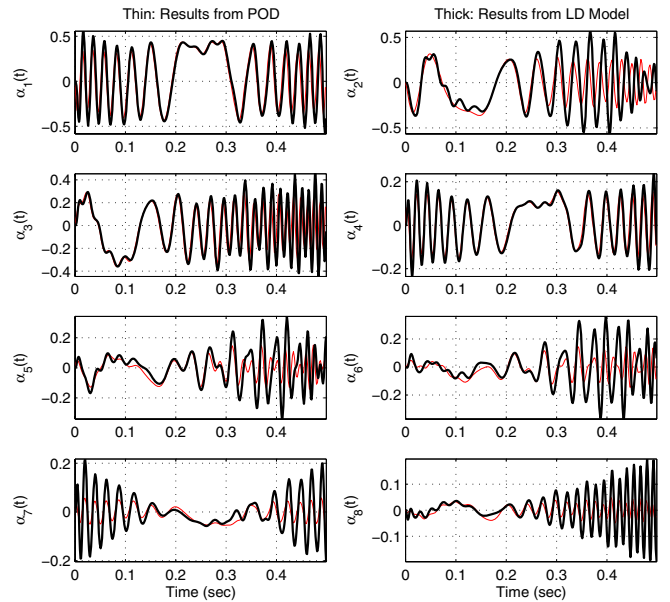

Fig. 2. The state variables $\left(\alpha_{k}(t)\right)$ obtained from POD and those from the LD model in (18).

$$
\begin{aligned}
\gamma_{1}(t) & =\sin (2 \pi 115 t(T / 2-t)), \\
\gamma_{2}(t) & =\sin \left(2 \pi 100 t\left(T / 4-t^{2}\right)\right), \\
\gamma_{3}(t) & =\sin \left(2 \pi 160 t\left(T / 2-t+t^{2}\right)\right), \\
\gamma_{4}(t) & =\sin (2 \pi 90 t(T / 5-t)),
\end{aligned}
$$

and obtained the results illustrated in Figure 5 and Figure 6. It is seen that the state variables are obtained precisely when the signal changes slowly. During the regions where the signals change quickly, the performance is relatively poor due to the spectral dependence of the model properties to the signals used during the derivation of the model, which are illustrated in the right subplots of Figure 1. To justify this claim, take the Laplace transform of the PDE in (1) and write the general solution as below;

$$
s \mathcal{U}(x, y, s)-u(x, y, 0)=c^{2}\left(\mathcal{U}_{x x}(x, y, s)+\mathcal{U}_{y y}(x, y, s)\right)
$$

where $u(x, y, 0)$ had been specified to be zero over $\Omega$. This would let us have the following general solution

$$
\mathcal{U}(x, y, s)=C_{1} e^{x \sqrt{s} / c}+C_{2} e^{-x \sqrt{s} / c}+C_{3} e^{y \sqrt{s} / c}+C_{4} e^{-y \sqrt{s} / c}
$$
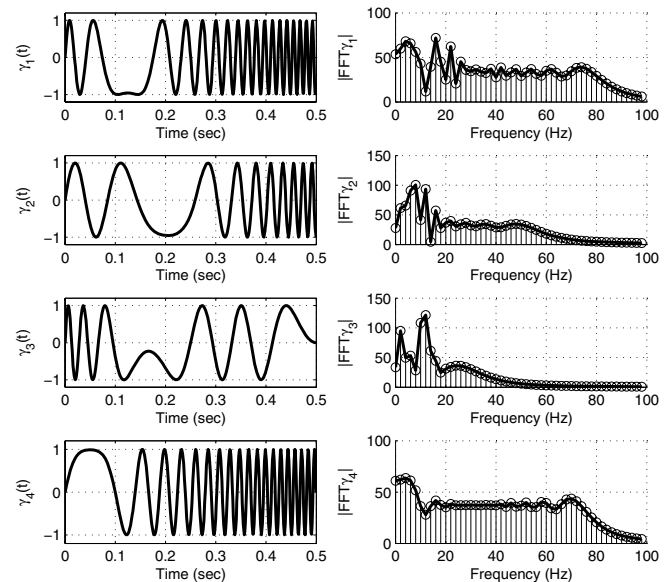

Fig. 3. Temporal and spectral views of the second set of boundary excitations.
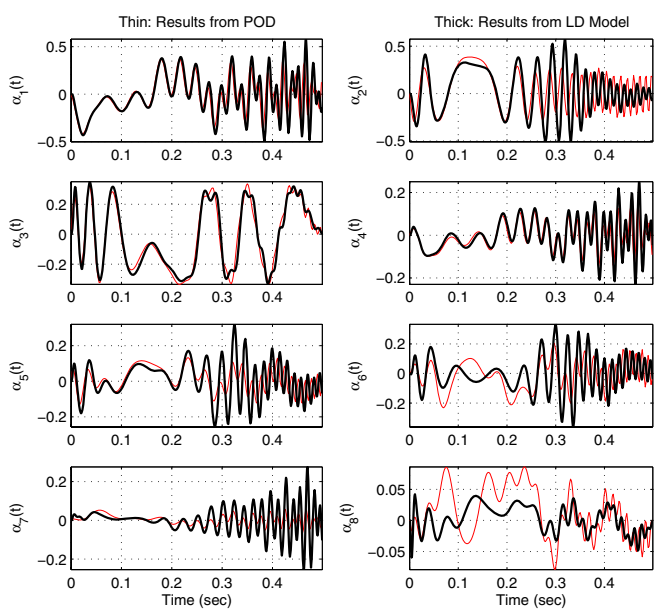

Fig. 4. The state variables $\left(\alpha_{k}(t)\right)$ obtained from POD and those from the LD model in (18) for the second set of boundary excitations.

Having postulated the solution, we obtain the following expression with irrational transfer functions

$$
\begin{aligned}
\mathcal{U}(x, y, s)= & \frac{\mathcal{N}_{1}(x, y, s)}{\mathcal{D}(s)} \Gamma_{1}(s)+\frac{\mathcal{N}_{2}(x, y, s)}{\mathcal{D}(s)} \Gamma_{2}(s)+ \\
& \frac{\mathcal{N}_{3}(x, y, s)}{\mathcal{D}(s)} \Gamma_{3}(s)+\frac{\mathcal{N}_{4}(x, y, s)}{\mathcal{D}(s)} \Gamma_{4}(s),
\end{aligned}
$$

where the details of the functions seen above are skipped due to the space limit.

Substitute $s=j \omega$ into (31). It becomes clear that once the external excitations are specified, the response $(\mathcal{U}(x, y, j \omega))$ is determined 

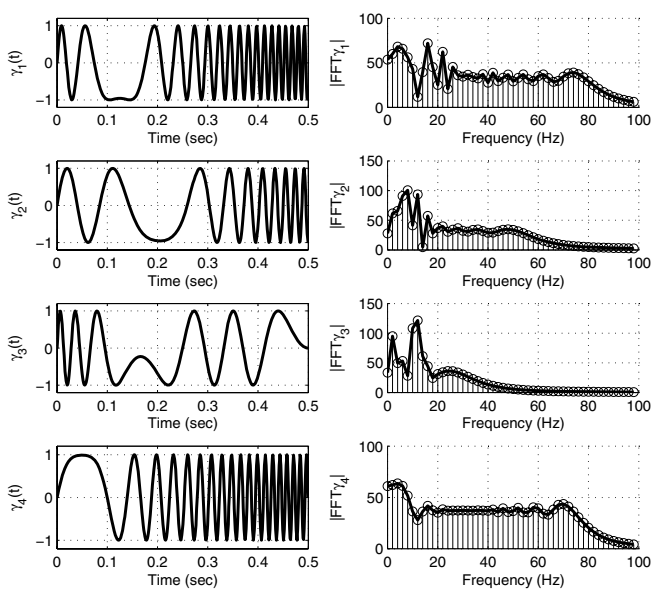

Fig. 5. Temporal and spectral views of the second set of boundary excitations.
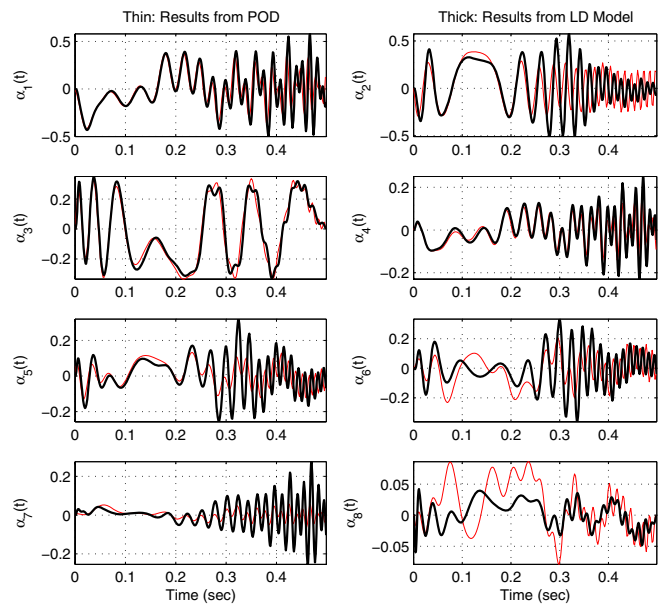

Fig. 6. The state variables $\left(\alpha_{k}(t)\right)$ obtained from POD and those from the LD model in (18) for the second set of boundary excitations.

by the transfer functions $\frac{\mathcal{N}_{i}(x, y, j \omega)}{\mathcal{D}(j \omega)}$ and the external excitations. The representation in (31) explains the conclusion on the spectral dependence of the LD model to the signals used during the model derivation stage. Nevertheless, one should keep in mind that the linearity of the PDE under investigation has facilitated achieving this conclusion. The next issue is to explain the nature of the mentioned dependence. A quick look at the spectral behaviors of the transfer functions $\frac{\mathcal{N}_{i}(x, y, j \omega)}{\mathcal{D}(j \omega)}$ stipulate that the magnitudes of these expressions has a lowpass characteristic with a cutoff frequency determined according to the value of $x$ and $y$. This tells us that for every $(x, y) \in \Omega$, the boundary excitations beyond the corresponding cutoff frequency will not affect the behavior at the point $(x, y)$, and the effect of such excitations are filtered out, i.e. their effect cannot be detected from the snapshots. A natural consequence is that those effects cannot be captured through the use of the snapshots in POD algorithm. On the other hand, the excitations below the cutoff frequency will be effective at the point $(x, y)$ and the snapshots will contain their effects as much as the $\frac{\mathcal{N}_{i}(x, y, j \omega)}{\mathcal{D}(j \omega)}$ 's permit. If the boundary signals are spectrally rich enough, then their effects will be reflected to the snapshots.

\begin{tabular}{|c|c|c|c|}
\hline$M=1$ & $M=2$ & $M=3$ & $M=4$ \\
\hline 19.31 & $17.92+j 4.96$ & 19.72 & $-61.59+j 247.96$ \\
- & $17.92-j 4.96$ & $21.92+j 14.69$ & $-61.59-j 247.96$ \\
- & - & $21.92-j 14.69$ & $22.07+j 13.19$ \\
- & - & - & $22.07-j 13.19$ \\
- & - & - & - \\
- & - & - & - \\
- & - & - & - \\
- & - & - & $\mathrm{M}=8$ (Stable) \\
\hline \hline $\mathrm{M}=5$ & $\mathrm{M}=6$ (Stable) & $\mathrm{M}=7$ (Stable) & $-197.44+j 616.26$ \\
$-61.43+j 244.47$ & $-59.78+j 224.47$ & $-148.51+j 491.47$ & $-197.44-j 616.26$ \\
$-61.43-j 244.47$ & $-59.78-j 224.47$ & $-148.51-j 491.47$ & $-148.37+j 491.18$ \\
$-66.60+j 275.43$ & $-61.62+j 257.24$ & -77.12 & $-148.37-j 491.18$ \\
$-66.60-j 275.43$ & $-61.62-j 257.24$ & $-75.26+j 311.68$ & $-59.65+j 236.47$ \\
20.98 & $-75.24+j 311.78$ & $-75.26-j 311.68$ & $-59.65-j 236.47$ \\
- & $-75.24-j 311.78$ & $-59.60+j 233.07$ & -59.67 \\
- & - & $-59.60-j 233.07$ & -76.88 \\
- & - & - & -93.21 \\
\hline
\end{tabular}

Fig. 7. Table of the eigenvalues of matrix $A$ as $M$ changes

Unsurprisingly the properties specified indirectly by the snapshots will be inherited by the LD model. As a result, the richer the boundary excitations spectrally the better the snapshots contain the spectral properties of the system dynamics. To sum up, the signals used in the modeling stage have significant effects on the performance of the LD model and those signals have to excite the system persistently in order to obtain a reasonably good model. This is one important contribution of this paper.

A final remark in this section is on the number of modes used. In this study, we have set $M=8$ modes in the model. The reason why we set such a value is related to the stability of the system in (18) as well as the reconstruction performance. In Figure 7 and Figure 8, we summarize the results obtained when $M$ starts from one and increases.

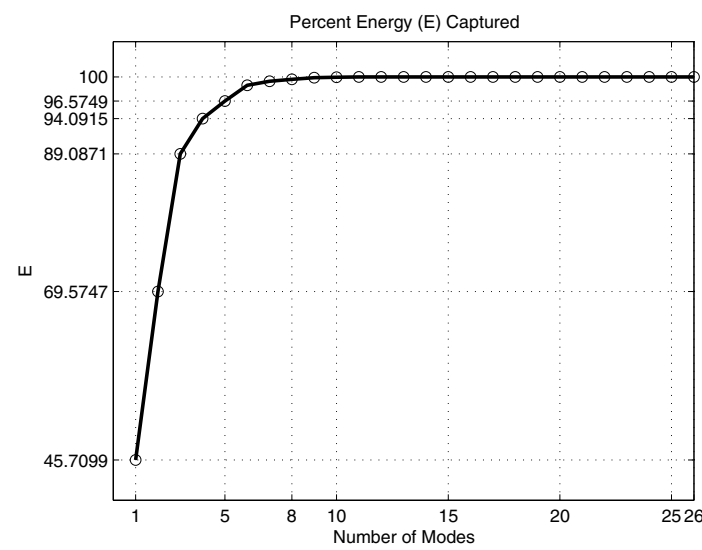

Fig. 8. The evolution of the percent energy captured with respect to the number of modes included

Figure 7 lists the eigenvalues of the matrix $A$ in (18) for $1 \leq$ $M \leq 8$. As seen from Figure 8, even though the captured energy level $(E)$ increases as $M$ is increased, the resulting system turns out to be unstable for $M \leq 5$, i.e. $E \leq 96.5749 \%$. A stable system with smallest number of modes is obtained for $M=6$ but the model performance is still not satisfactory and we need to increase $M$ further. When $M=8$, the percent energy measure indicate that the $\% 99.6563$ of the total energy is captured and the reconstruction performance of the model is good. This discussion stipulates two facts: first the stability of the reduced order model is not guaranteed for all values of $M$, second the concept of captured energy level is meaningful only in the close neighborhood of $E \approx \% 100$. In 
Figure 9, we illustrate the cases for every possible $M$ value. As seen clearly, the maximum of the real part of the eigenvalues of $A$ indicate stability for $M \geq 6$ and a convergence after $M=13$. Note that the observation of the convergence seen in Figure 9 further indicates the appropriateness of the numerical solver parameters such as $\Delta x, \Delta y$ and $\Delta t$. In what follows, we demonstrate the design of an observer for the PDE in (1).

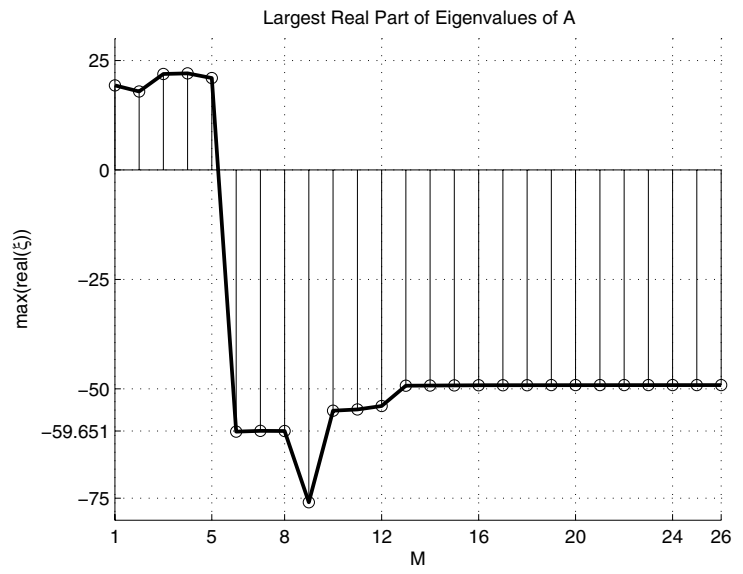

Fig. 9. Maximum of the real part of eigenvalues of matrix $A$ for every possible $M$ value.

\section{CONCLUSIONS}

This paper considers POD based LD modeling of 2D heat flow excited continuously along the boundaries. The paper validates the model and emphasizes that the model is useful over a set of operating conditions. The simulation results have shown that the selection of mode number and the associated tradeoff emphasizes the case specificity. Because of the linearity of the PDE process, the modal composition of the reduced order model could have been scrutinized and the weaknesses and strengths of the modeling strategy have been discussed in detail.

This paper has three major and two minor contributions to the subject area. One of the major contributions is on the choice of the mode number $(M)$ and its potential effects in control system design. The desire for obtaining a good match between the temporal variables from POD and those from the LD model, if it exists, may require utilizing more than a few modes. However, due to the associated numerical problems of POD, enriching the modal content can make the LD model vulnerable in terms of the structural properties such as stability and state controllability.

The second remarkable contribution is the demonstration of the spectral dependence of the LD model on the initial and boundary in the model derivation stage. If the design procedure entails a model that is to be used over a limited range of frequencies, then the PDE can be solved for boundary conditions which have dominant components in the range of interest. The paper unfolds that the choice of the external excitations are substantially important in terms of the LD model performance and this has a guiding nature in the implementation of POD since a very high level of captured energy does not imply a satisfactory performance by itself.

The last major contribution is the assessment of the importance of the fundamental assumption. The bottom-up design is strongly based on to what extent this assumption holds true. The facts stipulated in this paper show that as the number of modes in the model increases, more energy is captured and better approximation is obtained. Consequently, the confidence of the designer on the fundamental assumption gets increased and the model uncertainty becomes negligible, yet the model starts losing its usefulness in the increasing direction of $M$, which is a significant parameter in modeling studies exploiting the POD approach. Likewise, in (9), we substitute $u(x, y, t)=\sum_{i=1}^{M} \alpha_{i}(t) \Phi_{i}(x, y)$ into the governing PDE. Such a substitution is allowed if and only if the fundamental assumption is satisfied. This emphasizes that if the eigenvalues of the correlation matrix $L$ do not decay logarithmically, then one cannot truncate the expression comfortably as the total contribution of the remaining terms on the overall behavior is not negligible. This discussion demonstrates how critical the hold of fundamental assumption is.

Aside from the major results above, one of the minor contributions is the extension of a previously proposed approach from pointwise excitation to excitations along nonpoint subdomains, i.e. along the boundaries. The separation scheme lets us use the model not only for a predetermined boundary control regimes, (Efe 2003, Efe 2004), but also for a set of boundary excitations. The second minor contribution is on the better understanding of POD, which is achieved by choosing a linear system.

Needless to say, the linearity of the PDE has been exploited in drawing the above conclusions. These conclusions and the modeling strategy investigated in this paper advances the subject area to the clarification of the following fact: POD is a powerful technique but its usefulness depends upon the PDE in hand, problem settings and the associated operating conditions.

\section{Acknowledgments}

The author would like to thank Prof. Hitay Özbay, Prof. Mo Samimy, Dr. James H. Myatt, Dr. J. DeBonis, Dr. Marco Debiasi, Dr. Russell C. Camphouse, Dr. Peng Yan, Xin Yuan and Edgar Caraballo for fruitful discussions in devising the presented work.

\section{REFERENCES}

[1] Atwell, J.A. and King, "Computational aspects of reduced basis feedback controllers for spatially distributed systems," Proc. of the 38th Conf. on Decision and Control, pp. 4301-4306, Phoenix, Arizona, U.S.A. 1999.

[2] Atwell, J.A. and King, "Proper orthogonal decomposition for reduced basis feedback controllers for parabolic equations," Mathematical and Computer Modelling of Dynamical Systems, 33, pp.1-19, 2001.

[3] Caraballo, E., Malone, J., Samimy, M., and DeBonis, J., "A Study of Subsonic Cavity Flows: Low Dimensional Modeling," AIAA 20042124, June 2004

[4] Efe, M.Ö. and Özbay, H., "Low dimensional modeling and Dirichlét boundary controller design for Burgers equation," International Journal of Control, 77, 10, pp.895-906, 2004.

[5] Efe, M.Ö. and Özbay, H., "Proper orthogonal decomposition for reduced order modeling: 2D heat flow," Proc. of the IEEE Int. Conf. on Control Applications (CCA'2003), Istanbul, Turkey, June 23-25, pp.1273-1278, 2003.

[6] Farlow, S.J., Partial Differential Equations for Scientists and Engineers, Dover Publications Inc., New York, pp.317-322, 1993.

[7] Gügercin, S. and Antoulas, A.C., "A comparative study of 7 model reduction algorithms," Proceedings of the 39th IEEE Conference on Decision and Control, Sydney, Australia, December 2000.

[8] Lumley, J., "The structure of inhomogeneous turbulent flows," Atmospheric Turbulence and Wave Propagation, Nauca, Moscow, pp.166176, 1967.

[9] Ly, H.V. and Tran, H.T., "Modeling and control of physical processes using proper orthogonal decomposition," Mathematical and Computer Modelling of Dynamical Systems, 33, pp.223-236, 2001.

[10] Ravindran, S.S., "A reduced order approach for optimal control of fluids using proper orthogonal decomposition," Int. Journal for Numerical Methods in Fluids, 34, pp.425-488, 2000.

[11] Rowley, C.W., Colonius, T, Murray, R.M., "Model reduction for compressible flows using POD and Galerkin projection," Physica DNonlinear Phenomena, 189 (1-2), pp.115-129, 2004.

[12] Rowley, C.W., "Model reduction for fluids, using balanced proper orthogonal decomposition," International Journal of Bifurcation and Chaos, 15 (3), pp.997-1013, 2005.

[13] Sirovich, L., "Turbulence and the dynamics of coherent structures," Quarterly of Applied Mathematics, XLV, no. 3, pp. 561-590, 1987. 